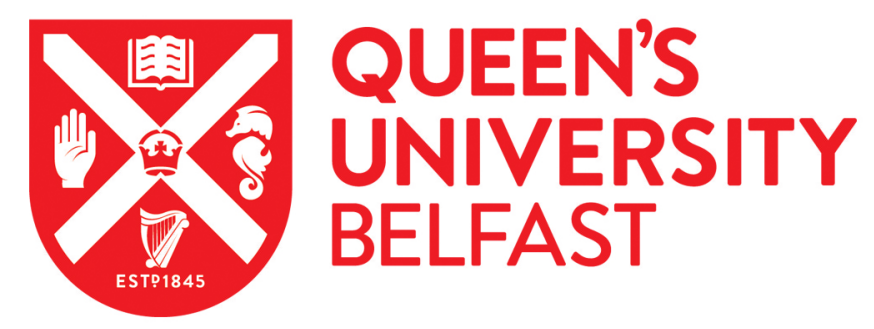

\title{
A Comparison of Online and Offline Strategies for Program Adaptation
}

Curtis-Maury, M., Antonopoulos, C. D., \& Nikolopoulos, D. (2007). A Comparison of Online and Offline Strategies for Program Adaptation. In Proceedings of the 45th Annual ACM Southeast Conference (ACMSE) (Vol. 2007, pp. 162-167). ACM. https://doi.org/10.1145/1233341.1233371

Published in:

Proceedings of the 45th Annual ACM Southeast Conference (ACMSE)

Queen's University Belfast - Research Portal:

Link to publication record in Queen's University Belfast Research Portal

\section{General rights}

Copyright for the publications made accessible via the Queen's University Belfast Research Portal is retained by the author(s) and / or other copyright owners and it is a condition of accessing these publications that users recognise and abide by the legal requirements associated with these rights.

Take down policy

The Research Portal is Queen's institutional repository that provides access to Queen's research output. Every effort has been made to ensure that content in the Research Portal does not infringe any person's rights, or applicable UK laws. If you discover content in the Research Portal that you believe breaches copyright or violates any law, please contact openaccess@qub.ac.uk. 


\title{
A Comparison of Online and Offline Strategies for Program Adaptation
}

\author{
Matthew Curtis-Maury ${ }^{1}$, Christos D. Antonopoulos ${ }^{2}$ and Dimitrios S. Nikolopoulos ${ }^{1}$ \\ ${ }^{1}$ Center for High-end Computing Systems ${ }^{2}$ Department of Computer Science \\ Department of Computer Science The College of William and Mary \\ Virginia Tech \\ $\{$ mfcurt,dsn\}@cs.vt.edu \\ and Greek Armed Forces \\ Division of Research and Informatics \\ cda@cs.wm.edu
}

\begin{abstract}
Multithreaded programs executing on modern high-end computing systems have many potential avenues to adapt their execution to improve performance, energy consumption, or both. Program adaptation occurs anytime multiple execution modes are available to the application and one is selected based on information collected during program execution. As a result, some degree of online or offline analysis is required to come to a decision of how best to adapt and there are a variety of tradeoffs to consider when deciding which form of analysis to use, as the overheads they carry with them can vary widely in degree as well as type, as can their effectiveness.

In this paper, we attempt to qualitatively and quantitatively analyze the pros and cons of specific types of online and offline forms of information collection and analysis for use in dynamic program adaptation in the context of high performance computing. We focus on providing recommendations of which strategy to employ for users with specific requirements. To justify our recommendations we use data collected from two offline and three online analysis strategies used with a specific power-performance adaptation technique, concurrency throttling. We provide a twolevel analysis, comparing online and offline strategies and then comparing strategies within each category. Our results show clear trends in the appropriateness of particular strategies depending on the length of application execution - more specifically the number of iterations in the program as well as different expected use characteristics ranging from one execution to many, with fixed versus variable program inputs across executions.
\end{abstract}

\section{General Terms}

Management, Measurement, Performance

Permission to make digital or hard copies of all or part of this work for personal or classroom use is granted without fee provided that copies are not made or distributed for profit or commercial advantage, and that copies bear this notice and the full citation on the first page. To copy otherwise, to republish, to post on servers or to redistribute to lists, requires prior specific permission and/or a fee.

ACMSE 2007, March 23-24, 2007, Winston-Salem, N. Carolina, USA

(C) Copyright 2007 ACM 978-1-59593-629-5/07/0003...\$5.00

\section{INTRODUCTION}

Although program adaptation is a relatively new area of research, many techniques have been proposed to allow an application to adaptively improve its execution time, energy efficiency, or both. Modern high-performance architectures contain many layers of parallelism, specifically processors, cores, and threads. These architectures leave much room for adaptation because it is not yet fully understood how best to exploit them given their inherent complexity. Further, it has been suggested that it is not possible to fully optimize an application without data collected during an execution of the program $[10,13]$.

Some of the more popular forms of adaptation are dynamic frequency and voltage scaling for energy, dynamically applying compiler optimizations for performance, and concurrency throttling for both. Each of these, and adaptation in general, can be performed by collecting data offline or online to make a decision on how best to adapt. Using offline analysis, data is collected during test program executions to make a decision that will then be used during the live runs. Alternatively, with online analysis, the data is collected during each live execution of the application, allowing for potentially different adaptation decisions for each run. Within these two general categories exist many specific collection and analysis techniques with their own advantages and disadvantages. Given the proven effectiveness of so many forms of adaptation, a thorough study of how best to find the optimal operating point under different circumstances is both timely and necessary.

To provide insight into general patterns in the tradeoffs of different analysis strategies, we have selected specific representatives from general categories. The details of the selected techniques are presented in Section 3, however in general we picked two offline and three online strategies to evaluate. To serve as our example adaptation technique we use concurrency throttling which adapts a multithreaded application to use fewer processors, cores, and hardware threads to reduce execution time and/or energy consumption. This is an especially pertinent optimization given the growing importance of energy-efficient computing.

The results collected from our experiments show clear trends in the effectiveness and overhead of general classes of analysis strategies. From this data we are able to make recommendations about which analysis technique is most appropriate for a particular user. We feel that our results are 
representative of most adaptation strategies and so our recommendations are applicable to many users.

In the next section, we present related work and provide background. In Section 3, we describe the adaptation technique used to evaluate the online and offline analysis strategies as well as the analysis strategies themselves. Section 4 gives our experimental results and our discussion thereof, including our recommendations. Finally, we conclude in Section 5 .

\section{BACKGROUND}

Previous work compares offline empirical-search versus offline model-based analysis within the compiler [16]. The purpose of this comparison is to determine whether adaptation of the form discussed in this paper is necessary at all. Their results show that using an accurate model for optimization of linear algebra code by ATLAS yields performance comparable to strategies employing empirical-search, with a significantly reduced offline overhead. However, the performance of the empirical-search approach is still better in most cases, and sometimes by a wide margin. This shows that program adaptation is still necessary, particularly in areas where highly accurate models do not yet exist.

There is a large body of related work on program adaptation and the work is largely split between techniques utilizing online and offline analysis strategies. In ATLAS [14], subroutines of BLAS (Basic Linear Algebra Suite) are optimized by executing each subroutine with many potential optimization parameters during offline test runs and selecting the one that performs the best for use online. Similar techniques are used in FFTW [6], SPIRAL [15], and PhiPAC [1].

Qasem et al. [11] propose an offline, profile-based analysis strategy for profitable application of loop fusion and tiling, which is difficult using compiler analysis alone as it is dependent on the underlying architecture and there are complex interactions between the two approaches. The offline analysis they perform is optimized to reduce the cost by using a realistic model to only test those configurations that are likely to perform well, rather than exhaustively searching all possible combinations. This is an effective strategy that limits the offline overhead. A similar model is used by Yotov et al. to reduce the offline search overhead of ATLAS [17].

One of the most popular forms of adaptation in the literature today is DVFS, or dynamic frequency and voltage scaling. Using this technique the processor frequency can be dynamically reduced to allow it to consume less power, while also slowing down the computations it performs. This enables an application to reduce its energy consumption without adversely affecting performance if it can be known that its performance is not limited by CPU frequency. DVFS has been used adaptively in work by Freeh, et al. [5] by sampling particular DVFS-levels live at runtime and using the one that best matches the desired execution characteristics. That work is later extended [8] to consider runtime performance prediction to decide DVFS-levels as well as the number of nodes in a cluster to use for execution.

Work on optimizing MPI calls at runtime has been presented by Faraj, et al. [4]. Since the same MPI routines are called many times during an application's execution, different algorithms can be tested for each routine. The authors perform the analysis of which algorithm to use at runtime, first with an exhaustive search and then an approach to heuristically search the algorithms to reduce the online overhead. Each MPI call site within the code can be adapted to use the optimal algorithm based on its properties.

Continuous Program Optimization [10] is a technique where a program is dynamically recompiled based on feedback collected at runtime, and the newly compiled image is hotswapped and used for execution. This process is then repeated for as long as the application is running. Kistler et al. [10] use this method for adapting object layout to improve cache locality and for dynamic trace scheduling to improve instruction level parallelism. While these optimizations could be performed using compiler analysis, the authors suggest that runtime information is necessary to achieve improved performance since program input information as well as user actions are only available at runtime.

There are many other examples of program adaptation, however those presented give a representative sample that exploit various types of online and offline analysis to determine optimal operating points.

\section{ADAPTATION STRATEGY}

To evaluate the various analysis strategies that can be applied to program adaptation in general in high performance computing, we chose one particular adaptation strategy, concurrency throttling, which we have discussed in previous work [2], to use for experimentation. Using this technique, an application can dynamically reduce the number of processors, cores, and hardware threads used for execution to simultaneously decrease both execution time and energy consumption. This approach can lower energy consumption by reducing the number of execution units actively drawing current. At the same time, performance can be improved because scalability bottlenecks that negatively impact performance can be reduced by using a lower degree of parallelism.

Analysis is needed to determine which hardware configuration will provide the desired properties, in terms of either performance or energy consumption. In this work, we consider adaptive concurrency throttling where the configuration with the highest expected performance is selected for use. Even with this form of concurrency control, power and energy consumption are both generally decreased due to the use of fewer processors in many cases. The decision making process requires knowledge of the execution times of the application running on different hardware configurations, as is also the case with other adaptation approaches. Different analysis strategies, however, have different pros and cons, and the purpose of this paper is to determine under what circumstances each technique excels.

In the following subsection we discuss the specific analysis techniques evaluated in this paper and how they relate to approaches presented in other work. The analysis techniques chosen are representative of larger classes of strategies, so the results are applicable to a wide range of approaches, beyond those specifically mentioned. 


\subsection{Offline Analysis Techniques}

The first two analysis techniques that we considered perform their analysis on data collected from offline test executions of the application. This class of analysis strategies is representative of all approaches where the work is done offline, rather than during the live execution. The advantage of these approaches is that they can search for optimal operating points without contributing to the execution time of the application online, however they can be expensive in terms of their offline overhead as they must be trained separately for each application.

The first offline strategy that we tested was offline_static, where the program is run once under each possible hardware configuration and the execution times are recorded. Then, the configuration with the lowest execution time is used for the live execution of the specific application. This is similar to the analysis strategy employed by Whaley, et al. [14].

The second offline strategy we used was offline_phases, where the adaptation occurs at phase granularity rather than statically for the whole application execution. Program phases are simply sections where execution properties remain fairly stable. Using this technique allows for potentially better performance because different phases within an application are likely to have varying execution characteristics, resulting in different optimal configurations [12]. The decision process for this approach is similar to that of offline_static except that the execution times of all offline test runs are recorded per phase, and the configuration with the lowest execution time for each phase is used online.

\subsection{Online Analysis Techniques}

In addition to the two offline strategies, we also selected three online analysis techniques. The first two of these work through live sampling of specific hardware configurations during runtime and the third works by making performance prediction based on a limited number of live test configurations. The online techniques exploit the iterative nature of parallel scientific applications by using the initial iterations of each phase as test executions on different configurations. All three techniques are phase aware.

\subsubsection{Empirical Search}

The first online strategy that we tested was online_exh. Using this approach, each phase of the application is run for one iteration under each hardware configuration during the live execution. Once all configurations have been tested, the observed optimal configuration is used for each phase for the remainder of execution. This search strategy requires $P * C * T$ test iterations during the live execution to come to a decision, where $P$ is the number of processors, $C$ is the number of cores, and $T$ is the number of hardware threads available in the system. An example from the literature of this type of analysis is presented in the STAR-MPI work of Faraj et al. [4].

We modified online_exh to reduce the number of hardware configurations that are tested online, rather than exhaustively searching all configurations, since there may be many configurations on modern architectures. This strategy, called online_heur, uses a hill climbing approach to first find the number of processors, then the number of cores, and finally the number of hardware threads to use for execution. At the beginning, the application is run with all execution resources, then along each dimension the resource is decremented by one until the execution time increases, executing each configuration for one iteration. This reduces the number of test configurations - and therefore iterations and online overhead - required to reach a decision to at most $P+C+T$. Further, the configurations that are eliminated from testing are likely to be those that perform the worst, specifically those with the fewest execution resources. This strategy is similar to the heuristic work done by Freeh, et al. [5] and Kistler et al. [10] to reduce the number of iterations required to find an effective configuration. Faraj et al. [4] also present a enhanced empirical search in their work on adaptively optimizing MPI collective operations.

\subsubsection{Performance Prediction}

To further reduce the number of iterations required to find an effective configuration, we have implemented and tested a performance prediction based approach, online_pred [2]. Here the optimal configuration is not found through active sampling of different configurations. Rather, we run the application on predefined hardware configurations and use information that is collected during the execution to predict the performance on other configurations. The predictions are then used to make the decision of which configuration to use. This technique is similar to the analysis approach used by Springer, et al.[8].

On architectures with multiple layers of parallelism, only one configuration is tested to make predictions along each layer. Specifically, the application is run with all execution resources active, and from this information predictions are made for different numbers of physical processors. Then this is repeated to decide the number of cores and again for hardware threads. Under this approach, only one test run is required for each layer of parallelism in the system.

A predefined set of performance counters are collected during each test iteration. The selected counters correspond to areas of processor activity that dominate performance in multithreaded codes, such as cache misses, bus transactions, etc. During offline training, performance counter values and execution times are collected for each of the training benchmarks on each hardware configuration. We then derive coefficients using regression to calculate the instructions per cycle - a standard numeric representation of performance that is often used for performance prediction - of each hardware configuration based on the observed performance counter values during the test runs of the training benchmarks. These coefficients can be applied to counter values collected online to predict the performance of any application at runtime.

\section{RESULTS AND DISCUSSION}

In this section, we describe our experimental methodology as well as the results of our experiments comparing various online and offline analysis strategies for program adaptation.

\subsection{Experimental Setup}

In our experiments, we used the OpenMP version of the NAS Parallel Benchmark Suite 3.1 [9] (Class A) which is a collection of scientific applications parallelized using the OpenMP 


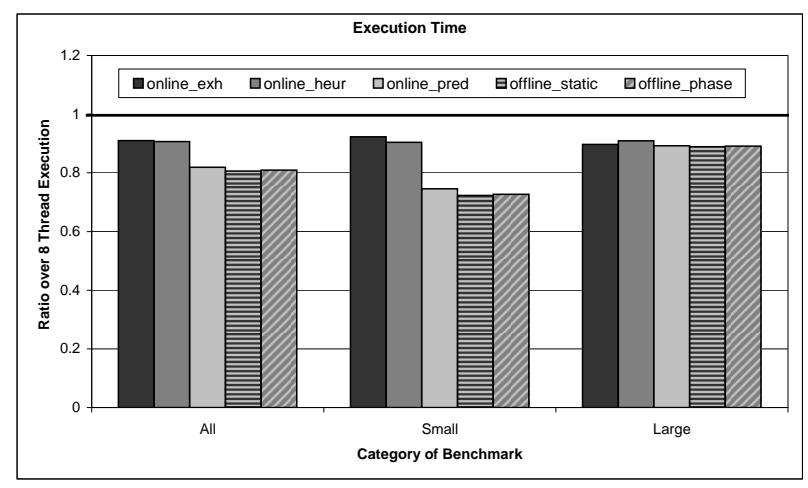

Figure 1: The execution times of each strategy used with concurrency throttling on different sets of benchmarks. Lower numbers are better.

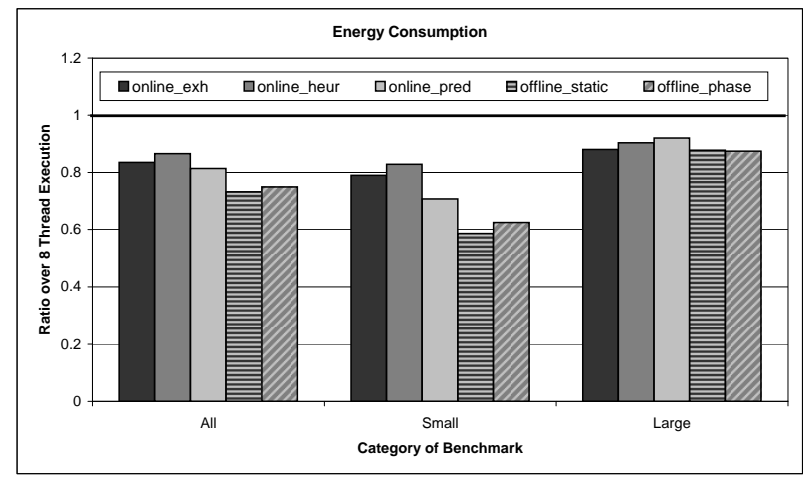

Figure 2: The CPU energy consumption of each strategy used with concurrency throttling on different sets of benchmarks. Lower numbers are better.

standard [3]. We ran our experiments on a Dell PowerEdge server composed of four Intel Xeon Hyperthreaded processors running at $1.4 \mathrm{GHz}$. The machine was running Linux kernel version 2.4.25.

We approximate phases with parallel regions in OpenMP. Parallel regions serve as appropriate estimates of phases as they are likely to have consistent properties during execution. Further, parallel regions are the finest granularity at which the number of running threads can be changed in OpenMP. For other application types, some alternative method of phase detection could be employed, such as the approaches presented by Sherwood, et al. [12].

In our analysis, we only present the energy consumption of the CPU, as that is our target for reduction. We estimate the CPU power consumption during each experiment using the approach presented by Isci, et al. [7]. This technique approximates power consumption by collecting performance counters to determine how active each unit of the processor is and it has been shown to be very accurate [7].

\subsection{Analysis of Results}

The results of our performance analysis are shown in Figure 1 and the CPU energy results are shown in Figure 2. All numbers are normalized with respect to the non-adaptive execution using all eight available execution contexts, which is the default behavior of a naive user. We refer to this strategy as naive. The left-most group of bars shows the results averaged over all benchmarks, the middle set of bars is the average results for benchmarks with a small number of iterations (15 or fewer in our evaluation), and the rightmost group of bars are averages for benchmarks with a large number of iterations.

Different applications require a different number of outermost loop iterations to reach a solution. For example $\mathrm{MG}$ from NAS requires only 4 iterations while SP requires 400 . We refer to applications with a large and small number of iterations as large and small applications, respectively. As applications with different numbers of iterations will benefit most from different strategies, due to varying amounts of online overhead, it is important to consider the results for the two application sizes.

Among the online analysis strategies, we begin by analyzing online_exh. This approach carries with it the largest overhead of the three online techniques as it must run the most test executions. Using this form of analysis results in a decrease in execution time by $9 \%$ and energy by $16 \%$ averaged over all benchmarks compared to naively using all available execution resources. These benefits are substantial, particularly since both metrics are simultaneously improved.

We next evaluate online_heur, our heuristic search. Using this technique the online overhead is greatly reduced by using fewer test executions. The relative improvement of this approach compared to online_exh will continue to increase on machines with more processors, cores, and hardware threads. In terms of performance, our results show online_heur outperforming online_exh by $2 \%$ overall, because it requires fewer iterations to come to a decision and therefore can spend more time in the decided optimal configuration. Despite the reduced execution time, energy consumption goes up by $3 \%$ because online_exh can find configurations that use fewer processors which online_heur may not test.

The strength of the heuristic search is its performance on applications with few iterations, where it outperforms the exhaustive search by $5 \%$. This gain can be explained by the fact that with few iterations, the applications are more sensitive to online overhead. On the other hand, for large applications online_exh is better by $1 \%$. The improved performance on large applications is due to their ability to amortize the increased overhead expense of a more thorough and effective search strategy.

The online_pred strategy further reduces the online search overhead by limiting the number of test executions even further, to only one per layer of parallelism on the machine. On our test machine with two layers of parallelism - processors and hardware threads - only two iterations are necessary. Using prediction based adaptation shows improvements of $18 \%$ in performance compared to naive, $10 \%$ better than online_exh and $9 \%$ better than online_heur. Further, the energy consumption is reduced by an average of $19 \%$ - better than either online_exh or online_heur. These two improvements occur because a decision is made in fewer iterations, so a larger percentage of execution is spent using configurations 


\begin{tabular}{|c|c|c|c|c|c|}
\hline & \multicolumn{3}{|c|}{ ONLINE } & \multicolumn{2}{|c|}{ OFFLINE } \\
\hline & EXH & HEUR & PRED & STATIC & PHASE \\
\hline offline overhead & 0 & 0 & $\begin{array}{l}\text { medium; } \\
\text { once ever }\end{array}$ & $\begin{array}{l}\text { small; } \\
\text { once for each app }\end{array}$ & $\begin{array}{l}\text { small; } \\
\text { once for each app }\end{array}$ \\
\hline online overhead & $\mathrm{P}^{*} \mathrm{C}^{*} \mathrm{~T}$ & $\mathrm{P}+\mathrm{C}+\mathrm{T}$ & $\mathrm{L}$ & 0 & 0 \\
\hline recommendations & $\begin{array}{l}\text { large apps; } \\
\text { few executions }\end{array}$ & $\begin{array}{l}\text { small apps; } \\
\text { few executions }\end{array}$ & $\begin{array}{l}\text { any size; } \\
\text { many different apps/inputs }\end{array}$ & $\begin{array}{l}\text { any size; } \\
\text { many times, one input }\end{array}$ & $\begin{array}{l}\text { any size; } \\
\text { many times, one input }\end{array}$ \\
\hline
\end{tabular}

Table 1: The offline and online overheads of each strategy $(\mathrm{P}=\#$ procs, $\mathrm{C}=\#$ cores, $\mathrm{T}=\#$ threads, $\mathrm{L}=\#$ layers of parallelism) and recommendations of appropriate times to use each strategy, based on application size and use patterns including how many times the application will be executed.

with better performance and lower energy consumption.

It is for small applications that the benefits of the reduced online overhead for online_pred truly appear. Compared to naive, prediction achieves a $25 \%$ speedup with a $29 \%$ reduction in energy consumption. Even compared to the other online analysis strategies for adaptation online_pred performs quite well for small applications, besting online_exh by $20 \%$ and online_heur by $16 \%$. For large applications, prediction is still very effective, achieving speedups of $10 \%$ compared to naive and $2 \%$ over the heuristic search, while matching the performance of the exhaustive search.

The results for the two offline search strategies were very similar to each other, showing a slight edge for offline_static. The explanation for the similarity is that while being phaseaware allows for the possibility of improved performance, offline_phase suffers from changing the configurations between phases, thereby hurting cache performance. However, for both application sizes, as well as overall, the two approaches see results within $0.5 \%$ of each other.

The performance of the offline analysis strategies is the best of any of the approaches tested. This is not surprising as the online overhead is reduced to zero when the work is done offline. Averaged over all benchmarks, the strategies improve performance by over $19 \%$ compared to naive, much better than the online empirical search strategies, but only $3 \%$ better than online_pred. Energy consumption is also reduced by $27 \%$ averaged over all benchmarks and $41 \%$ on benchmarks with few iterations.

The offline strategies are only slightly better than online_pred for small applications (by 5\%), but for large applications the results are comparable between the two approaches. In fact, for large applications the choice of analysis strategy is not especially significant as they all perform within $2 \%$ of each other - approximately $10 \%$ better than naive. This result shows that all of the techniques are capable of finding effective operating points, however they differ in their degrees of overhead. What separates the strategies for large applications is the amount of work required offline to use each. We address this issue in more detail in following subsection.

\subsection{Recommendations}

Having thoroughly compared the performance and energy consumption of each analysis strategy in the previous section, we are now in a position to analyze where each approach is most appropriate. Our recommendations, shown in Table 1, are based on the performance of each approach along with the offline overhead that must be endured by the users of each strategy. These tradeoffs suggest particular analysis strategies for users with different needs. Performance and energy results match, in general, and the recommendations for energy optimizing analysis strategies coincide with those for performance. This is true because increased online analysis increases both execution time as well as energy consumption since the selected configuration is being used for a smaller fraction of the total execution.

The online_exh and online_heur strategies have the desirable characteristic that their offline overhead, and therefore their cost to use, is near zero. The original code must simply be modified to make calls to the adaptation library before and after each phase to allow the adaptation to occur, which could be automated by a preprocessor. For this reason, these analysis strategies are particularly appropriate for users who only intend to run an application a small number of times. While the other strategies have better results, their offline overheads make them unsuitable for an application that will only be run once - or a small number of times - because the large offline overhead will not be amortized over many executions.

Within the category of applications that are run only a small number of times, online_heur shows itself to be the best candidate for small applications because it outperforms online_exh due to its reduced online overhead. On the other hand, the exhaustive empirical search yields better results on large applications, making it the best choice there.

For applications that will be run many times, a strategy that carries some offline overhead can be selected, because it will be made up for with the improved performance. The offline_static and offline_phase techniques are the best for applications that will be run many times, however only if the input is not changed across executions. The offline training techniques are tuned to the particular execution properties of an application and cannot adapt to them at runtime, so it is important that the offline training execution be identical to the live runs. However, changes in program input particularly input sizes - can lead to changes in the application's properties. As a result, offline training must be performed for each application as well as each different program input. Thus, offline strategies are limited in their applicability despite the fact that their performance is the best. They are, however, the most appropriate for precompiled libraries, such as ATLAS [14], that will be called many times. They are also appropriate for many users of scientific applications who execute the same application a large number of times. 
Between the two forms of offline analysis, we favor the use of phase-aware techniques like offline_phase. This is because phase-aware approaches have been generally shown to outperform static techniques [12], even if cache-effects prevent it from doing so for concurrency throttling.

Finally, online_pred has the largest offline overhead of the strategies evaluated. However, the overhead must only be paid once and prediction will work for any application of any problem size. The other online strategies also have this property, however online_pred outperforms these by a large margin on average. This advantage makes online_pred the most effective choice for applications of any size that will be run with different inputs or input sizes. Further, the training for prediction-based analysis works across applications, whereas the offline strategies require retraining for each application, so if multiple programs are to be run it is the best choice here as well. As online_pred works across applications and program inputs with a single offline training period, while still achieving comparable results to offline strategies, we feel that it is the best overall analysis strategy.

\section{CONCLUSIONS}

This paper has compared five different forms of analysis used for dynamic program adaptation. The specific techniques evaluated are representative of popular forms of analysis in the literature, and a thorough comparison of the strengths and weaknesses of each one was needed. The results of this study find that empirical search-based strategies are most effective when an application will be run a small number of times, due to the minimal offline overhead associated with their use. Alternatively, for applications that will be run many times with a fixed input, offline strategies are effective because they come with no online overhead. However, prediction-based analysis was shown to be the most effective approach for use with applications that will be run multiple times with different inputs or when multiple applications are going to be run with adaptation because the offline training must only be done once while its results were always within a few percent of the offline strategies.

The findings of this research are important because there has been an especially strong recent focus on finding ways of adapting applications at runtime to improve their performance or power. This interest has been due to the realization that only at runtime can full knowledge of an application become available. Our results are applicable beyond the domain of concurrency throttling and can be used both by researchers in the area of adaptation as well as users of adaptable systems.

\section{Acknowledgments}

This research is supported by the National Science Foundation (Grants CCR-0346867 and ACI-0312980) and the U.S. Department of Energy (Grant DE-FG02-06ER25751) and Virginia Tech. We would like to thank Henry P. Rose for his helpful suggestions on earlier drafts of this paper.

\section{REFERENCES}

[1] J. Bilmes, K. Asanovic, C.-W. Chin, and J. Demmel. Optimizing matrix multiply using PHiPAC: a portable, high-performance, ANSI C coding methodology. Proc. of the International Conference on Supercomputing, July 1997.

[2] M. Curtis-Maury, J. Dzierwa, C. Antonopoulos, and D. Nikolopoulos. Online power-performance adaptation of multithreaded programs using hardware event-based prediction. In Proc. of the 20th International Conference on Supercomputing, June 2006.

[3] L. Dagum and R. Menon. OpenMP: an industry standard API for shared-memory programming. IEEE Computational Science and Engineering, 5(1), 1998.

[4] Ahmad Faraj, Xin Yuan, and David Lowenthal. STAR-MPI: Self tuned adaptive routines for MPI collective operations. In Proc. of the International Conference on Supercomputing, June 2006.

[5] Vincent W. Freeh, David K. Lowenthal, Feng Pan, and Nandani Kappiah. Using multiple energy gears in MPI programs on a power-scalable cluster. In Proc. of the ACM Symposium on Principles and Practices of Parallel Programming, June 2005.

[6] Matteo Frigo and Steven G. Johnson. The design and implementation of FFTW3. Proceedings of the IEEE, 93(2), 2005.

[7] C. Isci and M. Martonosi. Runtime power monitoring in high-end processors: Methodology and empirical data. In Proc. of the 26th ACM/IEEE Annual International Symposium on Microarchitecture, November 2003.

[8] Robert C. Springer IV, David K. Lowenthal, Barry Rountree, and Vincent W. Freeh. Minimizing execution time in mpi programs on an energy-constrained, power-scalable cluster. In Proc. of the ACM Symposium on Principles and Practices of Parallel Programming, March 2006.

[9] H. Jin, M. Frumkin, and J. Yan. The OpenMP implementation of NAS parallel benchmarks and its performance. Technical report nas-99-011, NASA Ames Research Center, October 1999.

[10] Thomas Kistler and Michael Franz. Continuous program optimization: Design and evaluation. IEEE Transactions on Computers, 50(6), 2001.

[11] Apan Qasem and Ken Kennedy. Profitable loop fusion and tiling using model-driven empirical search. In Proc. of 20th International Conference on Supercomputing, June 2006.

[12] Timothy Sherwood, Erez Perelman, Greg Hamerly, and Brad Calder. Automatically characterizing large scale program behavior. In Proc. of the International Conference on Architectural Support for Programming Languages and Operating Systems, October 2002.

[13] Richard Vuduc, James W. Demmel, and Jeff A. Bilmes. Statistical models for empirical search-based performance tuning. International Journal of High Performance Computing Applications, 18(1), 2004.

[14] R. Clint Whaley and Jack Dongarra. Automatically tuned linear algebra software. In Proc. of the Supercomputing, November 1998.

[15] Jianxin Xiong, Jeremy Johnson, Robert Johnson, and David Padua. SPL: a language and compiler for DSP algorithms. In Proc. of the Conference on Programming Language Design and Implementation, June 2001.

[16] Kamen Yotov, Xiaoming Li, Gang Ren, Maria Garzaran, David Padua, Keshav Pingali, and Paul Stodghill. Is search really necessary to generate high-performance BLAS? Proc. of the IEEE, 93(2), 2005.

[17] Kamen Yotov, Keshav Pingali, and Paul Stodghill. Think globally, search locally. Proc. of the International Conference on Supercomputing, June 2005. 